\title{
Study of Factors Influence on the Variability of Time for Consensus Building in Coalitions Based on Regular Markov Chains
}

\author{
Olga V. Maksimova \\ Department of Global Climate Stabilization Research, \\ Yu. A. Izrael Institute of Global Climate and Ecology, Moscow, Russia. \\ E-mail: o-maximova@yandex.ru \\ Iosif Z. Aronov \\ Department of Commerce and Trade Regulation, \\ MGIMO (Moscow State Institute of International Relations) University, Moscow, Russia. \\ Corresponding author: izaronov@itandi.ru; aronoviz48@gmail.com
}

(Received on February 23, 2021; Accepted on May 7, 2021)

\begin{abstract}
Draft standard is approved if and only if consensus of the technical committee for standardization (TC) is ensured before its approval by the standardization body at the national, regional and international level. Previously published works of authors dedicated to the construction of a mathematical model of time to build a consensus (number of negotiations) in terms of variation in the number of TC members and their level of authoritarianism. The present study is a continuation of these works for the case of the formation of coalitions in TC. The mathematical model is constructed to ensure consensus on the work of technical standardization committees in terms of coalitions, which are overcome during the negotiation process by concessions. This paper analyzes the factors, which influence the time for consensus building in this case: the value of the assignment and an index of the effect of the coalition. It is proved that the time to build a consensus significantly depends on the value of unilateral concessions coalition and weakly depends on the size of coalitions with a fixed number of TC members. Built regression model of dependence of the average number of approvals from the value of the assignment. It was revealed that even a small concession leads to the onset of consensus, increasing the size of the assignment results (with other factors being equal) to a sharp decline in time before the consensus. It is shown that the assignment of a larger coalition against small coalitions takes on average more time before building a consensus.
\end{abstract}

The factors influencing the variability of the number of concessions were investigated. The change in the root-meansquare deviation (RMS) of the time to build a consensus varies nonlinearly from small concessions $Y=10 \%$ to large $Y=75 \%$, regardless of the ratio of the number of members between coalitions. In the simulations, at concessions $Y=10 \%$ there is the largest variation among the number of consensuses in the experiments.

Concession of the authoritarian leader in group reduces the number of negotiations and increases the quality of consensus. The results obtained are of practical value for all structures where the emergence of coalitions entails the impossibility of consensual decision-making and requires consideration of different ways to build a consensual solution.

Keywords - Technical committee for standardization, Consensus, Coalition, Regular Markov chains, Number of negotiations.

\section{Introduction}

This article intends to consider various details associated with building consensus in conditions of coalition formation in technical committees on standardization (TC). TC is an association of experts in a particular field, in which each draft of international standard (for example, ISO), regional standard (for example, CEN) or national standard (for example, GOST R) is examined before its approval by the standardization body. Standard is approved if, and only if, the consensus of the TC is ensured. 
National TCs are usually made up of representatives of scientific and public organizations, consumer associations and associations consisting of business representatives, as well as scientists and leading experts from organisations concerned. As of 2020, more than 240 TCs have been established in Russia, which review approximately 1000 national standards per year. The same approach to standards development has been adopted by virtually every country in the world, as well as at the regional and international level, for example, at the International Organization for Standardization (ISO).

Given the principle of the TC establishment, diverse interests when considering a draft standard, this often leads to coalition formation. Coalition formation is a dynamic process in TCs, because, initially, when considering the provisions of the draft standard, manufacturers may take one view of that subject, and consumers may take a different view, etc.

An analysis of the experience of participating in the ISO working groups responsible for the development of international standards has shown that coalition formation is very common in the development of standards. However, coalitions are normally breaking down during the negotiation process; otherwise, the number of adopted standards would be extremely small.

It should be stressed that the TC's work is evaluated in terms of the time to build consensus (Zazhigalkin et al., 2019), since in practice it is important to do it within the fixed period of time allotted for the examination. Therefore, the matter of assessing the time of convergence of the TC experts' opinions, depending on various factors that characterize experts' behavior during the negotiation process in the presence of coalitions in the $\mathrm{TC}$, is relevant.

The choice of research tools is determined by the specific form of the TC's work where participants tended to share common interests and tasks, where relationship is based on various trust levels, despite the presence of coalitions. This in turn does not allow to use the tool of the theory of games, including cooperative ones, in which the participants pursue their own (often antagonistic) goals in order to divide the payoff received by the coalition of agents (Shapley, 1971).

This is why the methods based on modeling agents' behavior in the formation of coalitions on the ground of the social choice and subjects' social dependence theories, allowing to solve the issues relating to the building of open dynamic coalitions, whose composition may change in the process of issue-solving, are not suitable for describing the negotiation process in the TC (Conte et al., 2001).

Models of agents' behavior in social networks, for example, also cannot better describe the activities of experts in the TC, merely because of fundamental differences between the latter and the social network: the stability of the TC structure, the lack of desire to increase the number of TC members (Gubanov et al., 2019).

Therefore, the mathematical tool of regular Markov chains, described by the authors in the following works (Aronov et al., 2018) was adopted as a consensus model, which proved its suitability for modeling in the case of a TC without coalition formation. In case of coalitions, the negotiation process is possible only with a concession made by at least one of the coalitions. With this as the background, a new task of developing and analyzing a model for building consensus with concessions made by coalitions is emerging, which is implemented herein. 
This article intends to study various factors at the time of consensus building and its variability within the constructed model with coalitions during the discussion of the provisions of the draft standard in the TC based on the modeling of regular Markov chains.

\section{Consensus Building Model Based on Regular Markov Chains}

Let's consider the model of the technical committee described in detail in (Zazhigalkin et al., 2019). Let $n$ be the number of experts participating in the discussion; $\mathrm{S}(0)=\left(\mathrm{s}_{0}{ }^{1}, \ldots ; \mathrm{s}_{0}{ }^{n}\right)-$ the vector of initial expert opinions, where $\mathrm{s}_{0}{ }^{i}$ is the opinion of the $i$ expert. The experts exchange opinions about the values of vector $\mathrm{S}$. Each expert's opinion may change in the process. Introducing the probability of confidence of the $i$ expert in the opinion of the $j$ expert through $0<p_{i j}<1(i=1 \ldots, n ; j=1 \ldots, n)$, we form a square trust matrix $\mathbf{P}_{n \times n}=\left(p_{i j}\right)$, which defines a sequential process of the opinions of TC members. The sum of the probabilities $p_{i j}$ in each row of the matrix equals 1 , i.e., for any $i \in \overline{1, n}$ the condition $\sum_{j=1}^{n} p_{i j}=1$ (matrix regularity) is satisfied.

Vector of expert opinions at each step is calculated by the formula:

$\mathrm{S}(1)=\mathrm{S}(0) \cdot \mathrm{P}_{n \times n}=\left(\mathrm{s}_{0}{ }^{1}, \ldots, \mathrm{S}_{0}{ }^{n}\right)$

After the $k$ step the vector of opinions is calculated by the formula:

$\mathrm{S}(k)=\left(\mathrm{s}_{k}{ }^{1}, \ldots, \mathrm{s}_{k}{ }^{n}\right)=\mathrm{S}(k-1) \cdot \mathrm{P}_{n \times n}=\mathrm{S}(0) \cdot \mathrm{P}_{n \times n}{ }^{k}$

Iteration process stops at the $m$ step if all rows of the matrix $\mathbf{P}_{\mathbf{n x n}}{ }^{m}$ are equal. Thus, the trust matrix $\mathbf{P}_{n \times n}$ reaches the final matrix F after $m$ iterations. Since the final matrix F does not change in subsequent iterations, the vector of expert opinions $\mathrm{S}(m)=\mathbf{S}(0) \cdot \mathbf{P}_{\boldsymbol{n} \mathbf{x} n}{ }^{m}=\left(\mathrm{s}_{m}{ }^{1}, \ldots, \mathrm{s}_{m}{ }^{n}\right)$ will not change either, i.e. consensus is built.

\section{Consensus Building Model in Coalitions}

The paper of authors (Zazhigalkin et al., 2019) studies the factors that influence the number of negotiations before the consensus is built, as well as the factors that influence the possibility of building it. Among the possible special cases in which the consensus is either delayed or not possibleare: domination, presence of multiple leaders, global domination, responsibility and coalitions shift.

It was noted that the formation of coalitions is a natural process due to the formation of the interests of different parties. Let's illustrate the mathematical model with two coalitions for a group of five members: expert 1 trusts himself and experts 2 and 3; expert 2 trusts himself and experts 1 and 3; respectively, expert 3 trusts himself and experts 1 and 2; group member 4 trusts himself and expert 5; expert 5 trusts himself and expert 4 . In this situation, two coalitions are observed, one including three experts and the other including two. One of the possible initial trust matrices $\mathbf{P}$ can have the following form:

$\mathbf{P}=\left(\begin{array}{lll|ll}0,6 & 0,2 & 0,2 & 0,0 & 0,0 \\ 0,4 & 0,5 & 0,1 & 0,0 & 0,0 \\ 0,2 & 0,3 & 0,5 & 0,0 & 0,0 \\ \hline 0,0 & 0,0 & 0,0 & 0,6 & 0,4 \\ 0,0 & 0,0 & 0,0 & 0,2 & 0,8\end{array}\right)$

From the theory of Markov chains (Gantmacher, 1959) we know that the necessary and sufficient condition of convergence of the initial matrix $\mathrm{P}$ to the final matrix $\mathrm{F}$ (the necessary and sufficient 
condition of building consensus) for any vector of initial opinions is regularity ${ }^{1}$ of matrix P. In other words, it is necessary and sufficient that sums of rows of matrix $\mathrm{P}$ are equal to 1 and the inequality $0<p_{i j}<1$ is satisfied for any rows for probabilities $p_{i j}$. In terms of the discussion of issues in a social group, it is important that some of its members have their own opinions and have confidence in the opinions of individual colleagues. The regularity condition is violated in the coalition model, so consensus is unattainable in this case. Matrices of this kind and their corresponding Markov chains are decomposable.

The established coalitions in TC can be eliminated by choosing compromise solutions. These solutions can be provided in different ways. Let's consider a situation in which there is a redistribution of trust probabilities $p_{i j}$ of the $i$ expert of one coalition to the $j$ expert of the second coalition, which is achieved by additional reasoning in the process of discussion. Such situation (redistribution of trust probabilities) will be called concessions.

This corresponds to the model outlined in the work of social psychologist Homans (1958). It proposes a scheme of functioning of small social groups (TC can be regarded as a small social group) on the basis of exchange of utilities.

Theoretically we can talk about two options for concessions:

Unilateral concessions in which members of one coalition redistribute their trust probabilities to members of a second coalition; ambilateral concessions in which members of one coalition redistribute their trust probabilities to members of the second coalition and, accordingly, members of the second coalition redistribute their probabilities to members of the first coalition.

Below there is a discussion about the analysis of the time required to build a consensus in unilateral concessions.

Unilateral concessions issues in the negotiation process have been addressed in a number of works, for example, in (Seungwoo and Weingart, 2004). However, they did not analyze the factors influencing time of the negotiation process. Note that the article (Kudish et al., 2015) concluded that the uniqueness of the conflict affects its time. This aspect is not considered in present article.

\section{Analysis of Coalition Influence Index and Value of Unilateral Concessions for Av- erage Consensus Building Time}

\subsection{Unilateral Concession by Members of Small Coalition}

Let's build a model of unilateral concessions on the example of two coalitions in a group of 20 group members. In the paper of Zazhigalkin et al. (2019), it is shown that this number of experts is optimal in terms of the number of negotiations before building a consensus under other equal conditions.

Let's assess convergence time of the opinion matrix $\mathbf{P}$ to the final matrix $\mathbf{F}=\mathbf{P}^{m}$. This time is determined by the required number of $m$ iterations (discussions) to form a consensus. The value $m$ will be calculated by matching all the corresponding column elements with two decimal places.

For simulation we have the following conditions: number of expert $n=20, m$ - the number of

\footnotetext{
${ }^{1}$ Matrices, which sums of elements of all rows are equal to one, are called stochastic. If for some $n$ all elements of the matrix $\mathbf{P}^{n}$ are not equal to zero, then such transition matrix is called a regular matrix.
} 
meetings before consensus is built (i.e., the time for consensus building), two coalitions. Let's introduce an index of coalitions influence $I$, equal to the ratio of number of members of the larger coalition to the smaller one. In practice, this can be interpreted as the quantitative "strength" of one coalition in relation to the other.

Simulation for case of unilateral concessions by members of a small coalition consisted of several stages. In the first stage, the levels of change in coalition's influence index I, were chosen:

1st: $I=4$ (coalitions with 16 and 4 members);

2nd: $I=1,5$ (coalitions with 12 and 8 members);

3rd: $I=1$ (in coalitions of 10 members each).

In the second stage, levels of concessions among members of a small coalition were selected. Numerous studies show that in small groups the group opinion is especially important, they are more consolidated (Gockel et al., 2008; Kerr, 1989), thus personal contacts allow for all members to participate in the development of group opinion and control of conformity of group members in relation to this opinion. Therefore, members of small groups are ready to consciously concessions equal in relation to its members for further conduct of negotiations. The following levels are distinguished for concessions of $Y$ :

$$
\begin{aligned}
& \text { 1st: } Y=10 \% . \\
& \text { 2nd: } Y=20 \% . \\
& \text { 3rd: } Y=33 \% . \\
& \text { 4th: } Y=50 \% . \\
& \text { 5th: } Y=75 \% .
\end{aligned}
$$

Each level specifies a percentage value for the small coalition, making concessions that is evenly distributed among the members of the other coalition. Here is an example of a transformation of the original trust matrix $\mathbf{P}$ for five experts with two coalitions ( 3 experts in the larger coalition and 2 experts in the smaller coalition) after concessions $Y=60 \%$ by members of the smaller coalition:

$\mathbf{P}=\left(\begin{array}{lll|ll}0,6 & 0,2 & 0,2 & 0,0 & 0,0 \\ 0,4 & 0,5 & 0,1 & 0,0 & 0,0 \\ 0,2 & 0,3 & 0,5 & 0,0 & 0,0 \\ 0,0 & 0,0 & 0,0 & 0,5 & 0,5 \\ 0,0 & 0,0 & 0,0 & 0,2 & 0,8\end{array}\right) \Rightarrow \widetilde{\mathbf{P}}=\left(\begin{array}{lll|lc}0,6 & 0,2 & 0,2 & 0,0 & 0,0 \\ 0,4 & 0,5 & 0,1 & 0,0 & 0,0 \\ 0,2 & 0,3 & 0,5 & 0,0 & 0,0 \\ 0,2 & 0,2 & 0,2 & 0,2 & 0,2 \\ 0,2 & 0,2 & 0,20,08 & 0,32\end{array}\right)(*)$

Actually, concession means that a coalition of two members "concedes" a probability of 0,6 , which is uniformly "passed on" to members of the larger coalition. We will count the number of concessions $m$ from this point onward.

For the considered matrix $\widetilde{\mathbf{P}}$ with number $m=6$ the final matrix $\mathbf{F}$ with accuracy of its elements $\varepsilon=0,01$ will have the form:

$$
\mathbf{F}=\left(\begin{array}{ccccc}
0,44 & 0,32 & 0,24 & 0,00 & 0,00 \\
0,44 & 0,32 & 0,24 & 0,00 & 0,00 \\
0,44 & 0,32 & 0,24 & 0,00 & 0,00 \\
0,44 & 0,32 & 0,24 & 0,00 & 0,00 \\
0,44 & 0,32 & 0,24 & 0,00 & 0,00
\end{array}\right)
$$


Value $\varepsilon=0,01$ for each $j$-th column of this $\mathbf{F}$ matrix determined the following absolute difference $\left|p_{i j}-p_{k j}\right|<\varepsilon$ for all rows $i, k(j, i, k=\overline{1,5})$.

Importantly, in the case of unilateral concessions, the consensus decision takes into account only the opinion of the coalition that accepts the concessions. Mathematically, this follows from the properties of matrices of the form (*) (DeGroot, 1974), but in practice it means that concessions reduce a party's own demands in order to allow the other party to achieve desired result.

At the third stage for each level $n$ modeling of elements $p_{i i}$ of matrix $\mathbf{P}$ was carried out using uniform law of distribution under given conditions of the first and second stages so that the sum of probabilities within each row equals 1, i.e., so that matrix $\mathbf{P}$ became stochastic. In order to obtain stable conclusions about the average number of $m$ variation of other parameters, 100 simulations were performed on each fixed level of factors $I$ and $Y$ in Excel (Efron and Tibshirani, 1991). The number was defined as the degree of matrix $\mathbf{P}$, at which in the final matrix $\mathbf{F}$ the elements within each column $j$ satisfied the condition $\left|p_{i j}-p_{k j}\right|<\varepsilon$ for all $i, k(j, i, k=\overline{1,20})$.

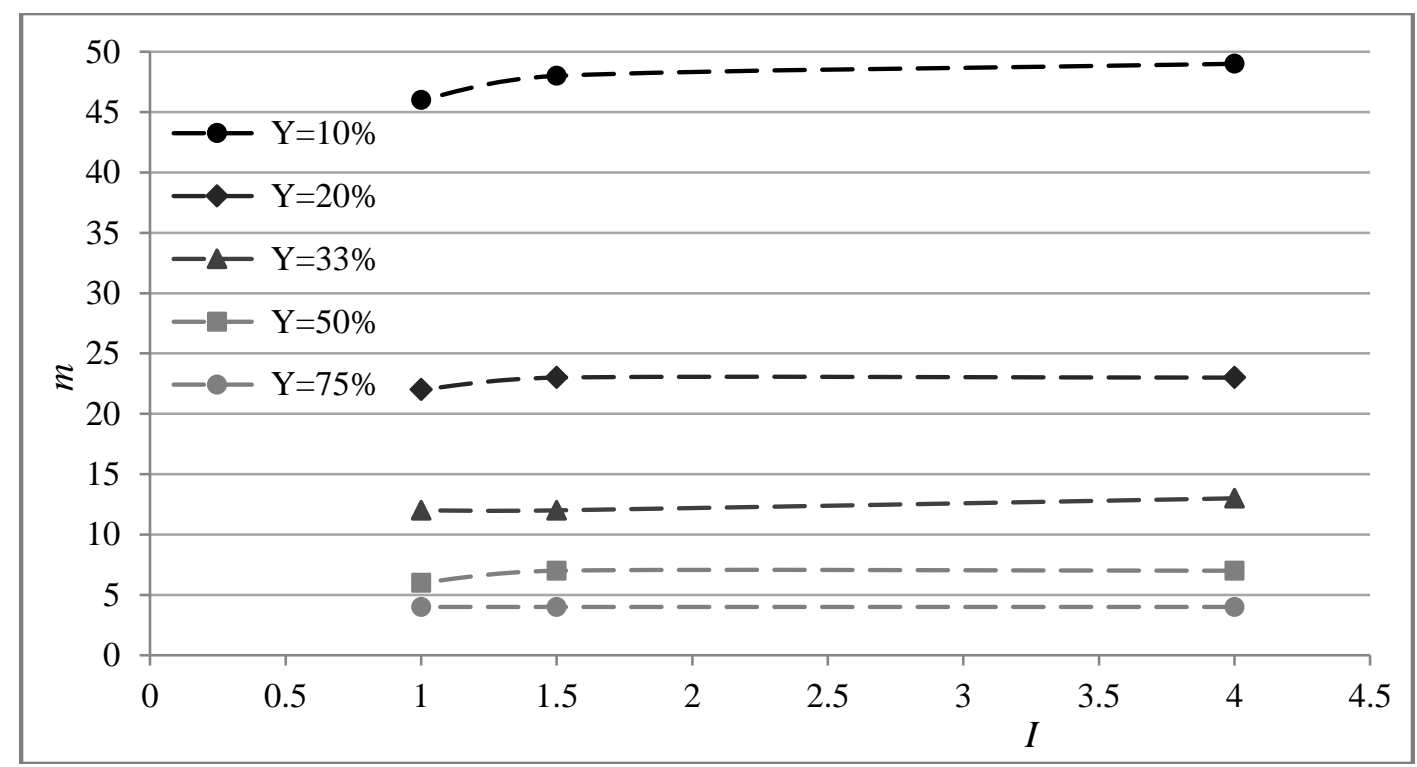

Figure 1. Dependence of the average number of negotiations $m$ on the coalition influence index $I$ at fixed levels of unilateral concessions $Y$ by members of a small coalition.

Figure 1 shows the dependence of the number of iterations (negotiations) $m$ on the influence index of the coalition $I$ at fixed levels of concessions $Y$. It turns out that at significance level $\alpha=5 \%$ the average values of number of negotiations at the level of concessions greater than $10 \%$ are statistically indistinguishable for different influence indices $I$. It can be concluded that the size of a large coalition has little effect on the number of negotiations before consensus is built.

For the average number of negotiations among different influence indices $I$ of coalitions a suitable regression is drawn, which has the form (Tukey, 1977).

$\widehat{m}=\widehat{a} \cdot Y^{\widehat{b}}$ 
where $\widehat{\boldsymbol{m}}$ - is the regression value of the number of iterations to build a consensus, $Y$ is the numerical value of concessions, $\widehat{\boldsymbol{a}}, \widehat{\boldsymbol{b}}$ are the equation factors.

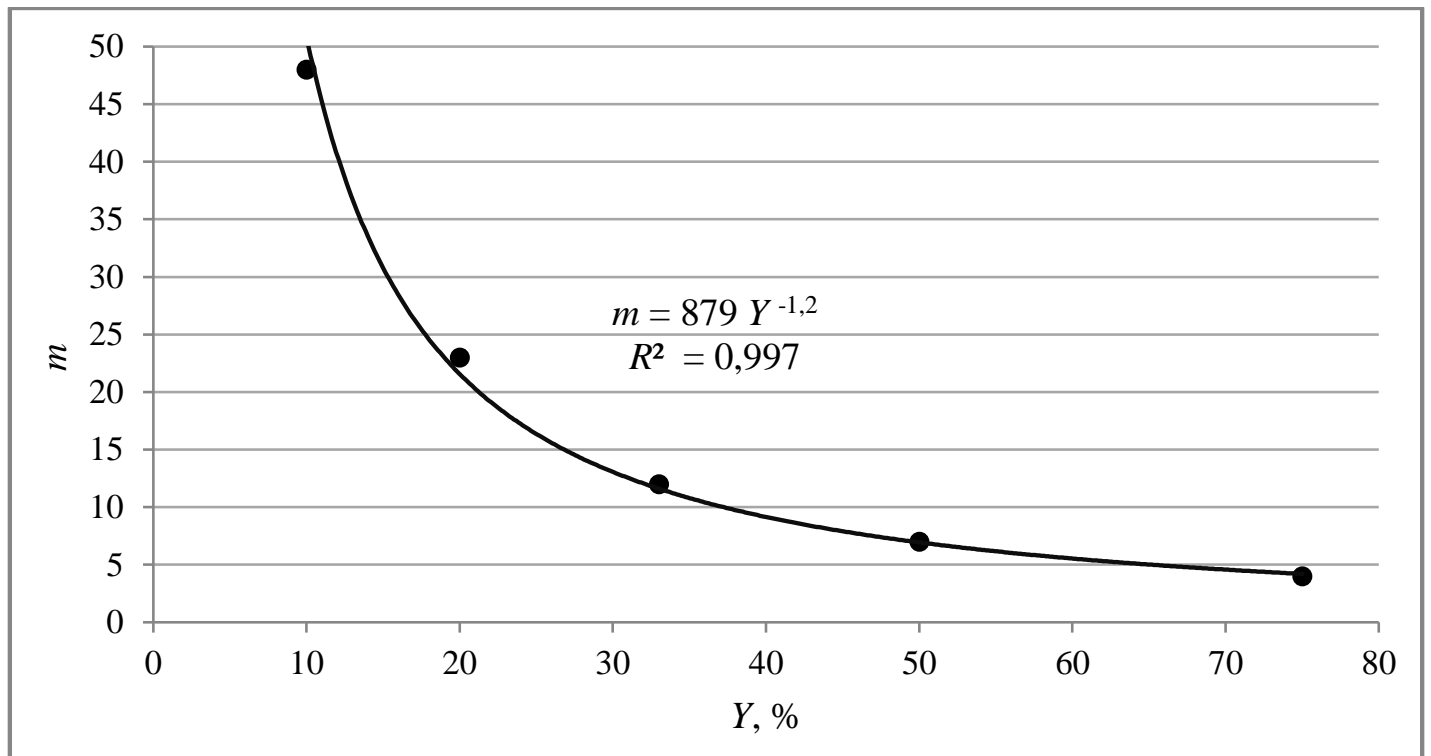

Figure 2. Dependence of the average number of negotiations $m$ from level of unilateral concessions $Y$ by members of small coalition (index of coalition influence $I=4$ ).

There is a gradual drop in the number of concessions with increasing value of concessions. Analysis of this model (2) confirmed not only visual, but also theoretical good negotiation with model data (for each curve $R^{2} \approx 0,997$, Figure 2 ). For example $\left(^{*}\right)$ at $Y=60 \%$, the average number of negotiations $m$ according to the calculated model is $m=879 \cdot 60^{-1,2} \approx 6$, which coincides with the experimentally obtained value in section 4 .

The diagrams in Figures 1 and 2 illustrate the high sensitivity of the $m$ number to the level of concessions $Y$ among the members of the small coalition, and the weak sensitivity for the index of coalition influence $I$.

Consensus is always achievable for the members of a small coalition. In this case, the lesser the concessions, the more time it takes to build a consensus. Even in small concessions, i.e., dedication for compromise, leads to a consensus. An increase in the number of concessions leads (among all other equal factors) to a sharp decrease in time before consensus (Aronov and Maksimova, 2020).

\subsection{Unilateral Concessions by Members of Large Coalition}

In section 1 it is found that the influence of the index I on the number of negotiations before the consensus is built is insignificant when the value of unilateral concessions is greater than $10 \%$. Consider the unilateral concessions model $Y=10 \%$ from the side of a large coalition and compare with the results of section 1. In order to obtain stable conclusions about the average number of $m$ concessions at each fixed level of the coalition's influence index, 100 simulations were performed in the Excel environment. The results presented in Figure 3 show that for small concessions $Y=$ $10 \%$ the fact which coalition makes it - small or large - is not important. In general, the more 
differences are observed in the number of two coalitions, the greater the difference in the average number $m$ before building a consensus (Aronov and Maksimova, 2020). However, these differences are statistically insignificant at the level of $\alpha=5 \%$ for all coalitions. At any index of coalition influence the significance of "strength" coalitions for convergence is leveled.

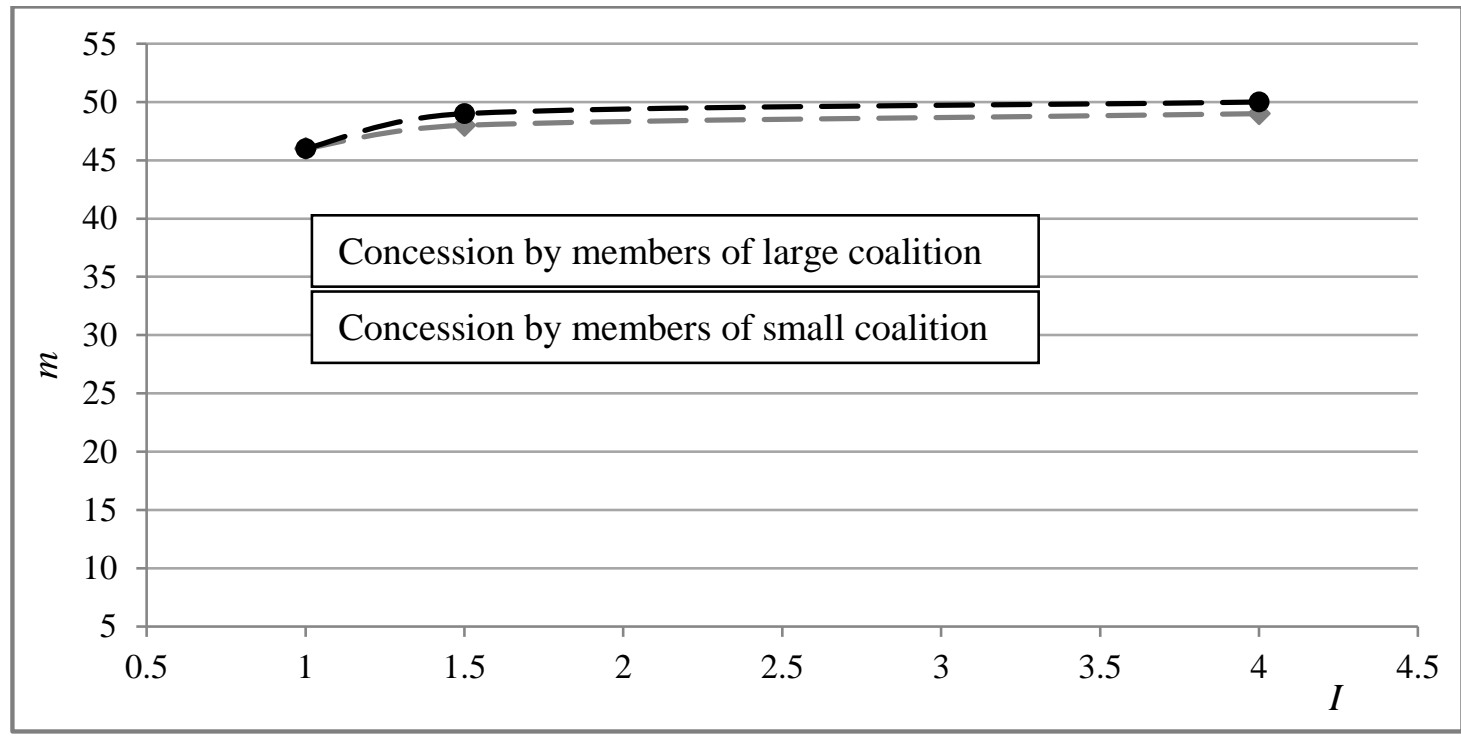

Figure 3. Dependence of the average number of negotiations $m$ from coalition influence index $I$, while unilateral concessions $Y=10 \%$.

In social groups this is confirmed by the way coalitions interact: in the case of concessions on the part of a small group, a large group "pulls over" the opinion of a small group by its "strength" (number of members), i.e., there is a quantitative nature of the process of negotiations.

When concessions are made by a large coalition, the reason for concessions can only be a strong argument for the position of the small group, because the opinion of the large coalition is not taken into account in the consensus decision. Thus, the consensus decision will be determined by the opinion of a small group, meaning, there is a qualitative character to negotiations.

In each of these methods the equal average number of negotiations before the consensus is built is provided by different nature of interaction between coalitions in group. Authors (Gockel et al., 2008; Kerr, 1989) in their papers came to the following conclusions:

small groups are more cooperative, rather than larger groups, and make better use of resources, available to them; one of the explanations for the difficulties of large groups is that, as the number of group members increases, each person's behavior becomes less identifiable, which is likely to lead to an increase in "stowaways". Large groups are more likely to have difficulties coordinating the efforts of individuals, and this may reduce quality of cooperation.

\subsection{Presence of Authoritarian Leader in Group}

Presence of authoritarian expert (for example, $p_{11}=1$ ) also describes the situation of two coalitions with number of members in 1 and $(n-1)$ - in the other. As follows from the paper (Aronov et al., 2018) consensus can be built only in conditions of trust for the authoritarian leader, however, for a 
rather significant number of iterations (negotiations), given that the authoritarian expert (if not conceded) always remains with his opinion, meaning that the authoritarian expert "pulls" other members towards his opinion. In other words, the quality of such consensus is relatively low. To increase the quality of consensus and decrease the time elapsed before its occurrence it would be reasonable for the authoritarian leader to make a concession. In this case the number of iterations is decreased significantly and the consensus-based decision turns out to be more balanced.

\section{Influence of the Unilateral Concession upon Variability of the Number of Negotia- tions}

At simulation of a consensus under the condition of coalitions, the issue of the influence exerted by various factors upon the average number of negotiations performed until the consensus was built, was considered in the previous sections. It is noted that different variability of the number of negotiations is observed under different conditions. Variability provides for an important understanding of the boundaries of variation of the number of negotiations in the coalitions, because there is no complete homogeneity inside a coalition. Therefore, it characterizes the constancy of the number of steps taken to build a consensus at fixed levels of the predetermined factors. If the variability is expressed strongly, it provides us with the reason to seek for new factors explaining the variability that would, in its turn, allow forming up the conditions for intragroup stabilization of the group members and further investigation of the model under the given conditions.

For the results of simulation described in section 4, Figure 4 provides the box-and-whiskers diagrams of the variability of the number of negotiations at the pre-defined parameters of the concession and the strength index of coalitions. Increasing of both interquartile scattering and the extremum is notable at transition from a large concession $Y=75 \%$ to a small one $Y=10 \%$ (Figure 4). A larger concession can be interpreted as a method for stabilization of the number of negotiations at different modes of simulation. In the case with a large concession all other possible factors characterizing the group do not result, at the pre-defined index $I$, in any essential variation of the time elapsing until the consensus is built.

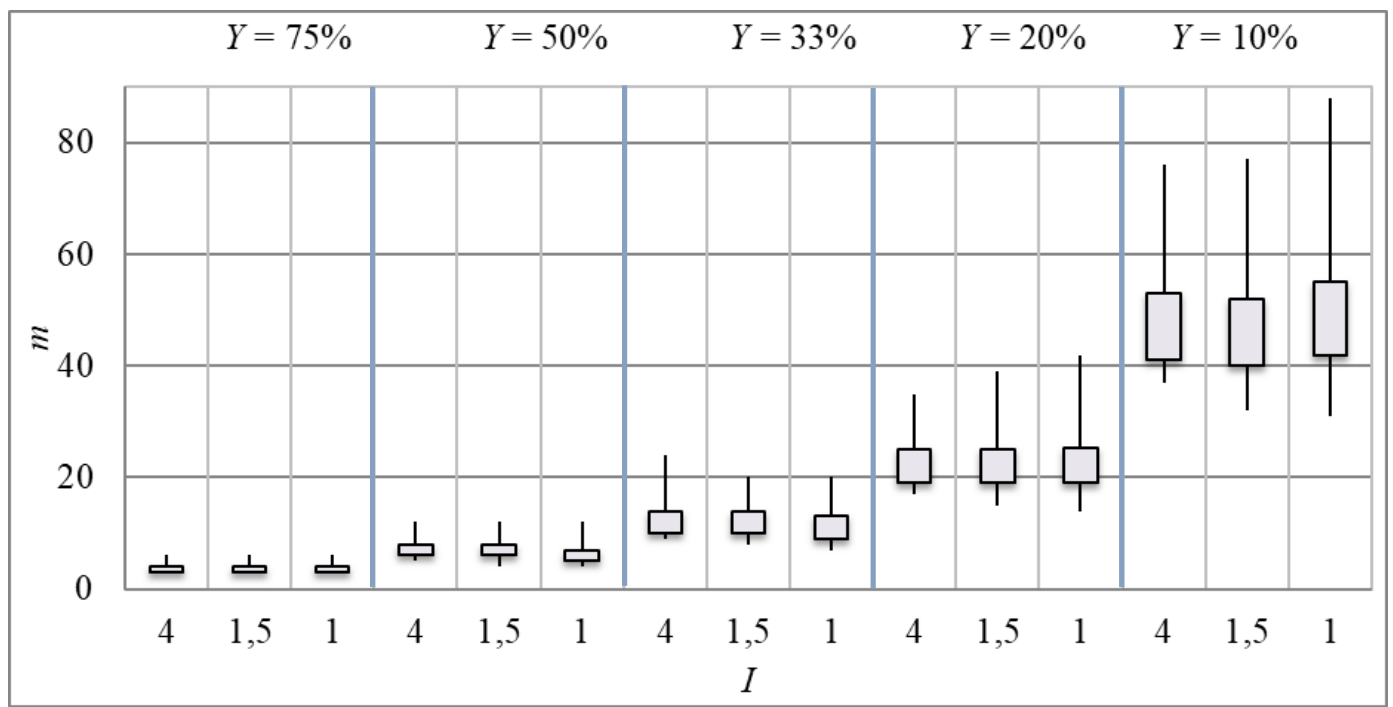

Figure 4. Box-and-whiskers diagrams for the number of negotiations $m$ at the pre-defined power index of coalitions $I$ and different concessions $Y$. 
At a small concession this stabilization is breached under all other equal conditions. Practically, it means that confidence in the number of negotiations $m$ predicted according to the formula (6) is lost for a specific group. Variation of the mean squared deviation (MSD) of the number $m$ occurs in a nonlinear manner from a small concession $Y=10 \%$ to a large one $Y=75 \%$ irrespective of the dependence on the ratio of the number of members between the coalitions $I$ (Figure 5). At a concession of $Y \geq 60 \%$ the value of MSD is $\leq 1$, i.e. there occurs stabilization of the number of negotiations from one simulation to the other.

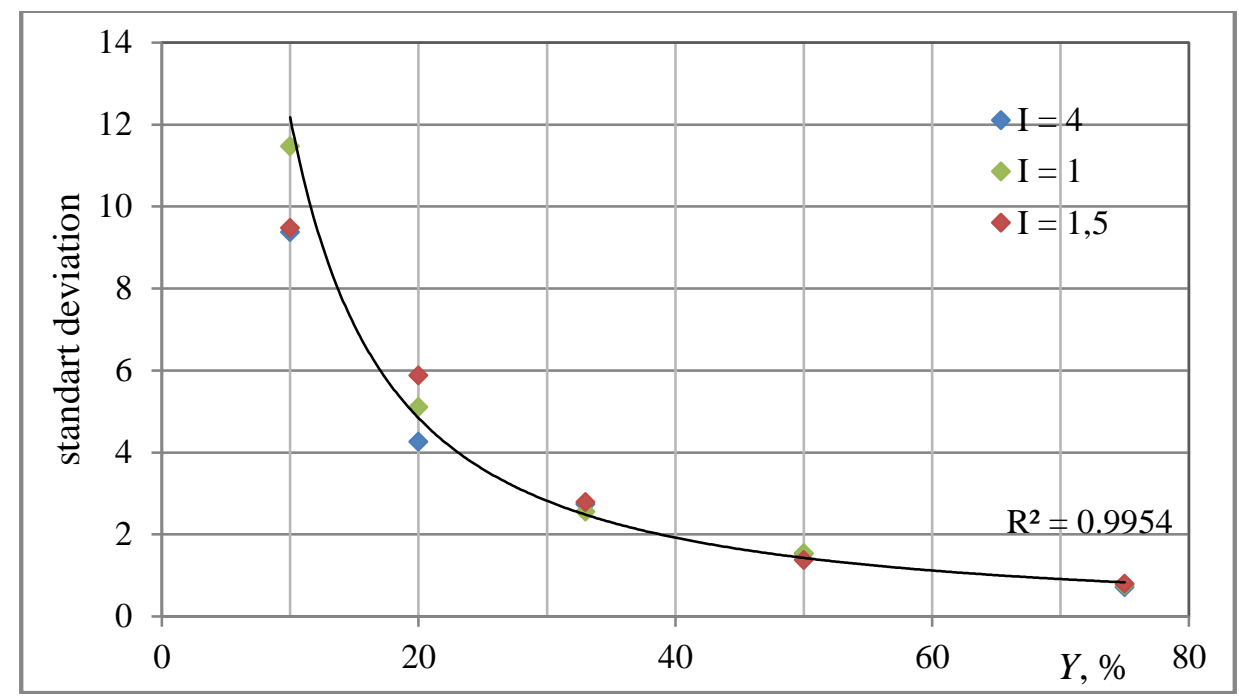

Figure 5. Dependence of MSD of the number of negotiations upon the concession $Y$ at different power indices of coalitions $I$.

During the simulation, the largest scattering among the number of negotiations in the experiments (Figure 5) is observed at the concession of $Y=10 \%$. It can be assumed that such variability is stipulated by interaction of the members both within each of the coalitions and between separate coalitions. Among all of the considered factors reflecting the structure of coalitions, the correlation analysis revealed a significant relationship $(r>0,5)$ of the number of negotiations with the maximum authoritarianism and the range of the confidence levels of the coalition accepting the concession.

In order to explain that variability in detail, the cluster analysis is performed in the Statistica 15 software module with the following assumptions (Figure 6): considering that the power index of coalitions $I$ exerts no influence upon the value of MSD, the value taken for the cluster analysis is $I=1$ that corresponds to the equal size coalitions; the concession is $Y=10 \%$; the clustering was performed based on three factors: 1) the number of negotiations $m, 2$ ) the highest authoritarianism $p_{i i}$ among the members of the coalition accepting the concession, 3 ) the range $R$ calculated among all the probabilities of the confidence of members of the coalition accepting the concession; the Euclidean distance was selected as the clustering metrics. 


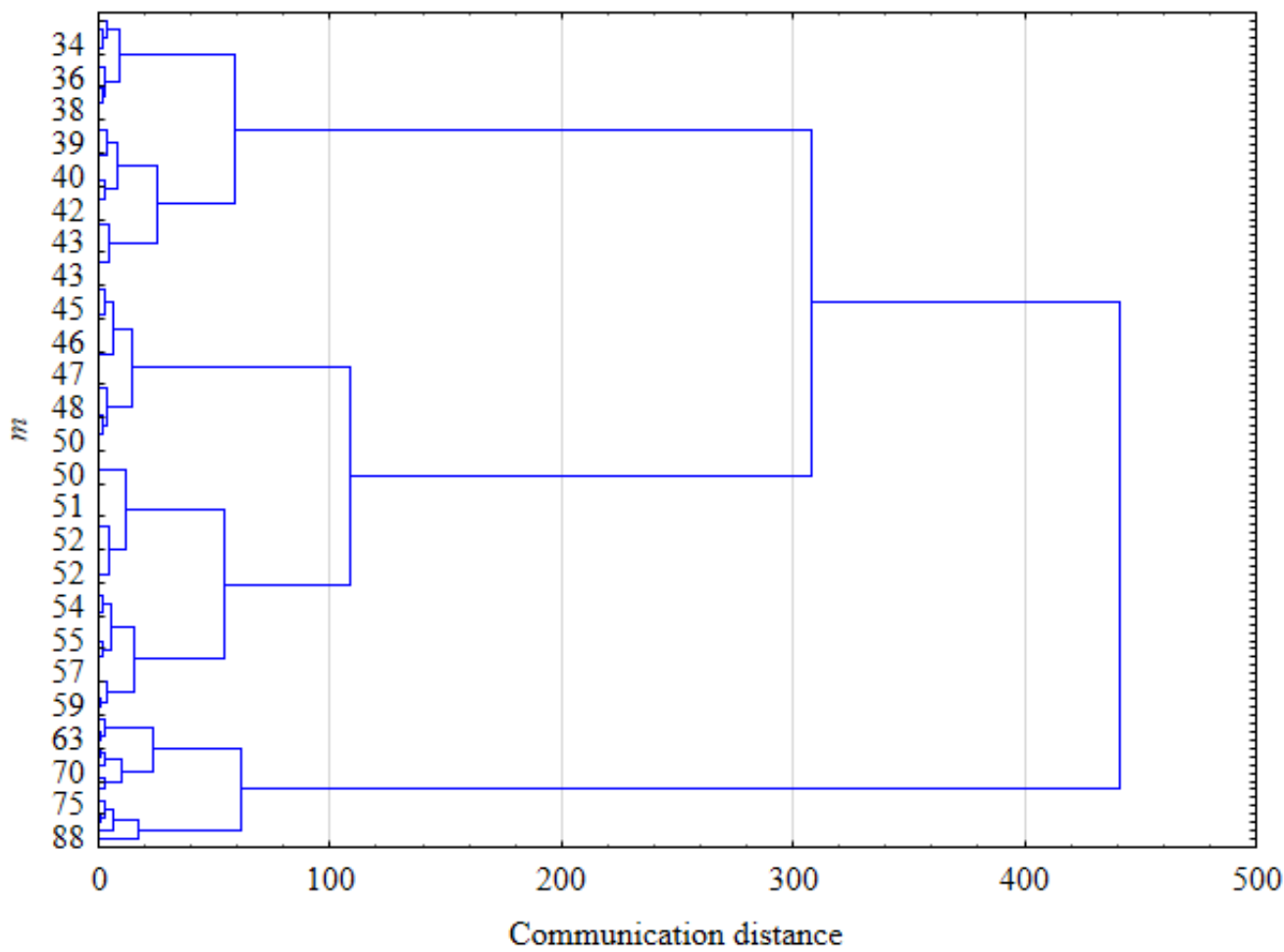

Figure 6. Cluster analysis for simulation of a consensus under the conditions of the equal size groups for a group of 20 members.

The highest values of the authoritarianism and range among the probabilities of confidence of the members of the coalition characterize the degree of negotiations of its members during the decisionmaking process. Considering that the consensus-based decision will be ultimately determined by just the opinion of the coalition, which accepts the concession (see section 4), then it is quite clear that it is its structure, which is of primary importance. The performed cluster analysis separated three large groups, in which the data are collected - with a small number of negotiations, a large and a medium number thereof (please see the values on the vertical axis in Figure 6).

Detail analysis of the structure of each cluster revealed the following interrelation: there exists a notable and significant correlational relationship $(r \approx 0,5 \div 0,6)$ within the clusters with a small (not more than 43) and a large (over 60) number of negotiations; for a medium number group that relationship turns out to be weak and insignificant - at the level of 5\%. It witnesses for the fact that the structure of the coalition (authoritarianism of its members $p_{i i}$ and the range $R$ of probabilities of the confidence of members of the coalition in the matrix $\mathbf{P}$ ), which accepts a small concession, exerts an influence upon the number of negotiations performed up to building a consensus. The more homogeneous it is (that is, the less the range and authoritarianism of the leaders are), the faster, on average, under all other equal conditions, the consensus will be built. It is matched with the result obtained at studying the factors influencing upon the number of negotiations for the technical committee without forming of coalitions (Aronov et al., 2018). 


\section{Conclusions}

A general conclusion that can be drawn from the results of simulation is reduced to the fact that the consensus can always be built at a unilateral concession. Whereas, the less value of the concession is, the more negotiations will be required to build a consensus. Even a small concession, i.e. striving to find a compromise, results in occurrence of a consensus. Increasing of the compromise size results (under all other equal conditions) in a sharp decrease of the time elapsing until the consensus is built.

It is shown that the time elapsing until the consensus is built is poorly dependent on the "strength" of the coalition. Whereas, a concession of a larger coalition in relation to a less numerous coalitions does not require more time to elapse until the consensus is built than otherwise. Practically it is stipulated primarily by a different mode of interaction between the coalitions in the group at different types of the concession: at a concession from a small coalition, a larger coalition "pulls over" the less numerous one due to a larger number of the members, while in order to force a large coalition to make a concession it would be necessary that a small coalition would provide a strong argumentation of its position. It is demonstrated that eigen demands of the party are decreased while making a concession in order to provide the other side with the opportunity to attain the desired result.

In the case when the coalition consists of one authoritarian leader, in order to increase the quality of consensus and decrease the time elapsing until the consensus is built, it would be reasonable for the authoritarian leader to make a concession. In this case the consensus-based decision turns out to be more balanced.

Studied variability of a number of negotiations until building a consensus showed that the structure of the coalition accepting a small concession exerts an influence upon a number of negotiations until building a consensus. The more homogeneous the coalition is, the faster, on average, the consensus will be built under all other equal conditions. At a larger concession stabilization of the number of negotiations occurs, and the initial structure accepting the concession loses its significance for the number of negotiations.

It is noteworthy that based on the known references social psychologists failed to study the time spent for search of the consensus under the conditions of coalitions ${ }^{2}$; therefore, the obtained results might be rather interesting for this sphere of research.

The obtained results can be distributed upon any and all organizational units, in which the decisions are made based on building a consensus: creation of coalitions in such organizations results in impossibility of the decision-making process within the framework of building a consensus and requires consideration of various methods used for taking a consensus-based decision.

Conflict of Interest

The authors confirm that there is no conflict of interest to declare for this publication.

With the only note that coalitions impede building a consensus. 
Acknowledgments

The authors would like to thank the editor and anonymous reviewers for their comments that help improve the quality of this work.

\section{References}

Aronov, I.Z., \& Maksimova, O.V. (2020). Theoretical modeling consensus building in the work of standardization technical committees in coalitions based on regular markov chains. Computer Research and Modeling, 12(5), 1247-1256.

Aronov, I.Z., Maksimova, O.V., \& Grigoryev, V.I. (2018). Analysis of consensus-building time in social groups based on the results of statistical modeling. In: Ram, M., Davim, J.P. (eds) Advanced Mathematical Techniques in Science and Engineering. River Publishers, Netherlands, pp.1-30.

Conte, R., Edmonds, B., Moss, S., \& Sawyer, K. (2001). Sociology and social theory in agent based social simulation: a symposium. Computational and Mathematical Organization Theory, 7(3), 183-205.

DeGroot, M.H. (1974). Reaching a consensus. Journal of the American Statistical Association, 69(345), 118121.

Efron, B., \& Tibshirani, R. (1991). Statistical data analysis in the computer age. Science, New Series, 253(5018), 390-395. http://www.jstor.org/stable/2878880?origin=JSTOR-pdf.

Gantmacher, F.R. (1959). The Theory of Matrices. AMS Chelsea Publishing, 660.

Gockel, C., Kerr, N.L., Seok, D.H., \& Harris, D.W. (2008). Indispensability and group identification as sources of task motivation. Journal of Experimental Social Psychology, 44(5), 1316-1321.

Gubanov, D.A., Novikov, D.A., \& Chkhartishvili, A.G. (2019). Social networks: models of information influence, control and confrontation. Springer, Switzerland.

Homans, G.C. (1958). Social behavior as exchange. American Journal of Sociology, 63(6), 597-606.

Kerr, N.L. (1989). Illusions of efficacy: the effects of group size on perceived efficacy in social dilemmas. Journal of Experimental Social Psychology, 25(4), 287-313.

Kudish, S., Cohen-Chen, S., \& Halperin, E. (2015). Increasing support for concession-making in intractable conflicts: the role of conflict uniqueness. Peace and Conflict: Journal of Peace Psychology, 21(2), 248263.

Seungwoo, K., \& Weingart, L.R. (2004). Unilateral concessions from the other party: concession behavior, attributions, and negotiation judgments. Journal of Applied Psychology, 89(2), 263-278.

Shapley, L.S. (1971). Cores of convex games. International Journal of Game Theory, 1(1), 11-26. https://doi.org/10.1007/BF01753431.

Tukey, J.W. (1977). Exploratory data analysis. Reading. MA: Addison-Wesley, (Vol. 2, pp. 131-160).

Zazhigalkin, A.V., Aronov, I.Z., Maksimova, O.V., \& Papic, L. (2019). Control of consensus convergence in technical committees of standardization on the basis of regular markov chains model. Springer India: International Journal of Systems Assurance Engineering and Management, 10(1), 29-36. 